\title{
Ecology and Diversity of Cyanobacteria in Kuttanadu Paddy Wetlands, Kerala, India
}

\author{
Dhanya Vijayan', Joseph George Ray ${ }^{2 *}$ \\ ${ }^{1}$ Environment Science Research Lab, St Berchmans College, Changanacherry, India \\ ${ }^{2}$ School of Biosciences, Mahatma Gandhi University, Kottayam, India \\ Email: ${ }^{j}$ jgray@mgu.ac.in
}

Received 28 September 2015; accepted 20 November 2015; published 24 November 2015

Copyright (C) 2015 by authors and Scientific Research Publishing Inc.

This work is licensed under the Creative Commons Attribution International License (CC BY). http://creativecommons.org/licenses/by/4.0/

(c) (i) Open Access

\begin{abstract}
Algae are one of the major groups of soil microflora in agricultural lands. Among algae, the bluegreens are considered to be very valuable in agriculture. The role of them in soil fertility enhancement has been extensively studied worldwide. Sustainable utilization of an organism for any human purpose depends on how successfully the ecology of the same is thoroughly understood. Kuttanadu is a unique tropical paddy-wetland. Ecology of blue-green-algae and the exact diversity of the same in the zone remained unexplored. This is the first report of the blue-green-algal community of Kuttanadu in relation to different soil-regions, seasons, and crop-growth-stages. A rich blue-green-algal diversity of 64 species, with Oscillatoriales as the dominants (38\%), is observed in these paddy-fields. The highest values for all the ecological parameters analyzed were found in the Lower Kuttanadu soil region, during Virippu season, at panicle stage of the crop whereas the lowest values for most of the parameters were observed in Upper Kuttanadu soils during puncha season at the seedling and panicle stages. The species richness and diversity index showed positive correlation to crop seasons. Apart from the specific soil and climatic factors, the total number of blue green algal isolates showed positive correlation to total nitrogen and phosphorus in the soils.
\end{abstract}

\section{Keywords}

Blue Green-Algae, Paddy-Wetland, Seasons, Soil-Regions, Growth-Stages

\section{Introduction}

Nitrogen deficiency is a common problem of rice soils worldwide. Several species of blue-green-algae or cyanobacteria with nitrogen fixing capacity are currently used as bio-fertilizer, especially to wetland paddy, in many

${ }^{*}$ Corresponding author. 
parts of the world [1]. Cyanobacteria as a fertilizer supplement are capable of replacing 30 - 40 percent of urea nitrogen requirement to paddy and they have other positive roles in soil management [2]. Role of cyanobacteria in paddy fields is a much explored theme of research [3]-[9]. Since most blue-greens are cosmopolitan species with wide ecological amplitude to occupy diverse environmental conditions, exploration of the diversity and ecology of them from specific paddy zones has global relevance towards the development of sustainable paddy farming everywhere in the world. Moreover, as they are photosynthetic species, quantitative assessment of them in such soils will be significant to the accounting of global carbon sequestration potentials of paddy soils in general. These algae are also suggested as solution to soil pollution [10]. Their roles in soil crust formation and surface stabilization [11], soil genesis, soil conservation [12], general soil fertility enhancement [13] are well known. Some algae are also known as agents of producing plant growth promoting substances [14]. Very many specific reports of cyanobacteria from paddy fields of Kerala region are available [15]-[18], but those of specific zones of Kuttanadu fields are quite rare [19] [20] and pretty old.

In these contexts, a comprehensive evaluation of the ecology and diversity of blue-greens of Kuttanadu rice fields is carried out. Major objectives included isolation and identification of all the specific species of bluegreens in the paddy fields of Kuttanadu, Kerala, South India in relation to soil regions, seasons and cropgrowth-stages. Quantitative ecological aspects of the entire cyanobacterial community in terms of population dynamics and relative abundance in relation to soil pH, Nitrogen (N), Phosphorus (P), Potassium (K), Calcium (Ca), and Magnesium (Mg) from three different soil regions of Kuttanadu in relation to two crop seasons (puncha and virippu) and two growth stages of paddy (seedling and panicle stage) are compared and analyzed. As such, this is the pioneer attempt to explore the ecology and diversity of blue-green-algae in Kuttanadu wetlandpaddy soils.

\section{Materials and Methods}

Kuttanadu $\left(90^{\circ} 17^{\prime} \mathrm{N}\right.$ to $90^{\circ} 40^{\prime} \mathrm{N}$; $760^{\circ} 19^{\prime} \mathrm{E}$ to $\left.760^{\circ} 33^{\prime} \mathrm{E}\right)$ is a unique tropical flooded paddy land of global interest situated in the South India Figure 1. It is the traditionally well known rice bowl of Kerala, spread around the Vembanadu Ramsar zone with a size of approximately $25 \mathrm{~km}$ in east-west direction and $60 \mathrm{~km}$ in north-south, direction, spread over 54 revenue villages of the three districts of Kerala State. This inland backwater zone had an estuarine character; but the construction of the "Thannirmukkam-barrage" during 1970s to protect paddy cultivation in the area from saline incursion during the non-monsoon season has caused the entire zone to become an artificially maintained freshwater system. These paddy fields lie at an average of 2 to $2.5 \mathrm{~m}$ below the mean sea level and remain freshwater-flooded all throughout the year except during the crop seasons when water is pumped out from the fields.

Major paddy crop seasons of the zone are Puncha (the summer crop-December to March) and Virippu (the monsoon crop-May to October). In general, the soil of Kuttanadu is highly compressible dark brown "alluvial" clay of high organic and specific mineral constituents [21]-[23] with slight variations over soil regions. Upper Kuttanadu, Lower Kuttanadu (Kari soils) and Kayal-lands are the three major soil regions of Kuttanadu [22].

On the basis of geological features and biochemical characteristics, soils of Kuttanadu are grouped into three categories such as Karappadom (found mostly in the upper Kuttanadu), Kari (mostly lower Kuttanadu) and Kayal (mostly in Kayal lands) soils. Karappadom soils spreads over a large part in the upper Kuttanadu covering an area of about 33,000 hectares. They are river-borne alluvial soils. They are located more towards the interior and exhibit salinity less than that of the Kari and Kayal soils. They are deep and poorly drained soils. Soil colour varies from dark grey to deep brown or black. The surface soils are generally clay loams and characterized by high acidity and a fair amount of decomposing organic matter and nitrogen. They are generally deficient in available plant nutrients, phosphorus and lime.

Kari soils are characterized by acidity and salinity, which are deep black in colour due to relatively higher proportion of organic matter. They are seen in both the upper and lower Kuttanadu regions. They are peat soils found in large isolation patches in Alappuzha and Kottayam districts covering an area of about 9000 hectares. They exhibit characteristics of submerged and burned mangrove forest area, but are not silted up. Soils are characterized by heavy texture, poor aeration, bad drainage and low content of available plant nutrients. The natural formation of sulphuric acid is reported in Kari soils. Free sulphuric acid is formed by the oxidation of sulphur compounds present in the wood fossils under the soil. Large amounts of woody matter at various stages of decomposition occur embedded in this soil.

Kayal soils are found in rice fields reclaimed from the Vembanadu lake beds at the Kottayam and Alappuzha 


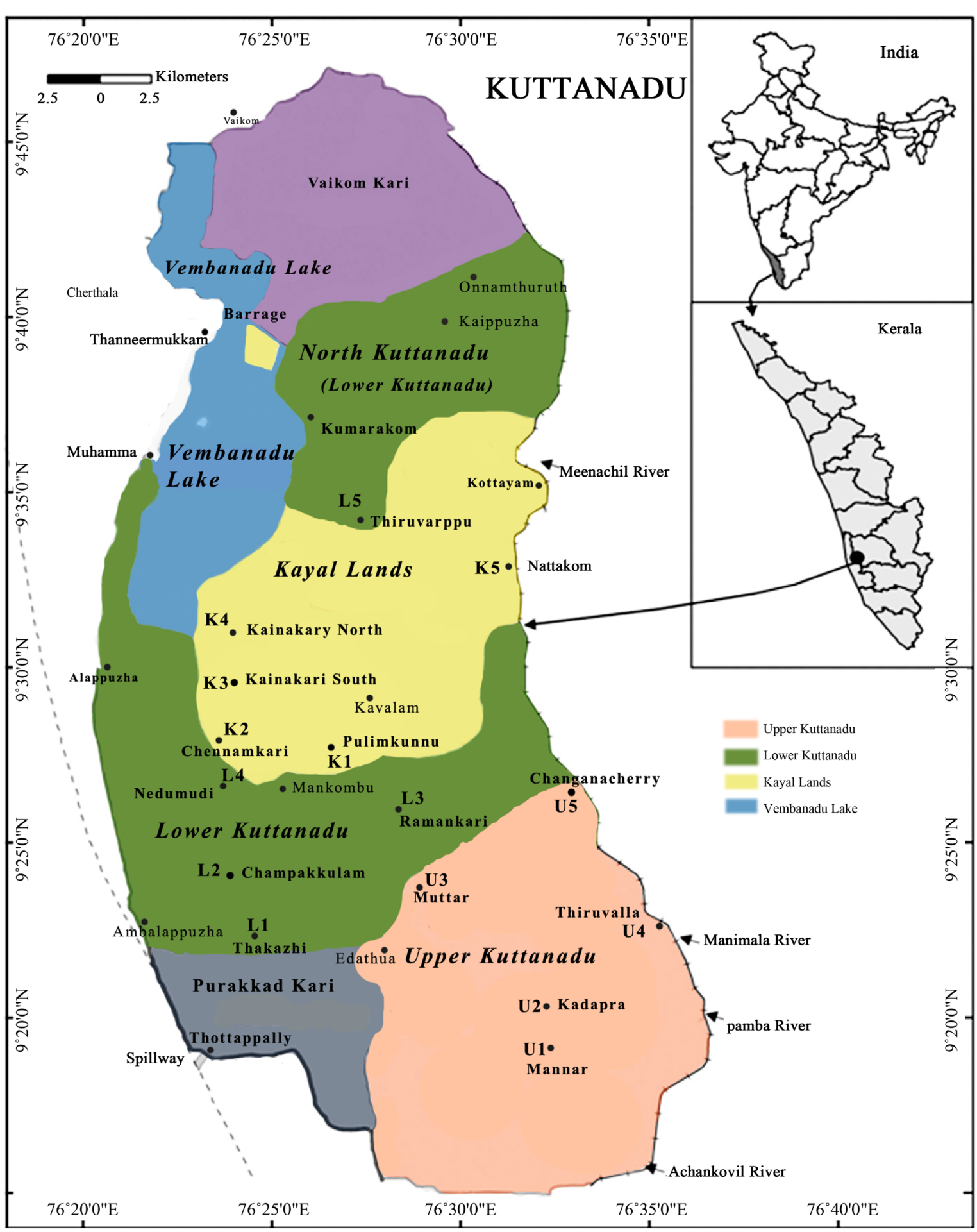

Figure 1. Map of Kuttanadu.

districts and occupy an area of about 13,000 hectares. They are deep and poorly drained soils with a dark brown colour. The texture varies from silt loam to silty clay loam. They are slightly acidic to neutral in reaction. They are low in inorganic matter content, poor in total and available plant nutrients, but are fairly rich in calcium.

In all tables R1 represents the Upper Kuttanadu, R2 the Lower Kuttanadu and R3 the Kayal Lands; S1 represents the Pre-monsoon (Puncha) crop and S2 the Monsoon crop (Virippu); G1 represents the seedling 
growth stage and G2 the panicle stage.

\section{Soil Sampling}

Separate soil samples were collected simultaneously for algal studies and physico-chemical analyses of soils. All the analyses were carried out using standard Methods. Soil sampling for blue-green-algae was the same as that for soil and green algal studies of the zone already reported [23]. Soil sampling was carried out from five different paddy fields located about- $2 \mathrm{~km}$ intervals for each of the three soil regions. Two different composite soil samples of $1-5 \mathrm{~cm}$ depth were collected from each paddy field. At each field, samples were collected from several random plots of $10 \mathrm{~m}^{2}$ size and mixed them together to a composite soil sample. Altogether 40 different composite samples (5 fields $\times 2$ plots $\times 2$ crop seasons-Puncha and Virippu $\times 2$ crop-growth stages-seedling stage and panicle stage) were collected from a soil region. Parallel to soil sample collection for physico-chemical analyses, surface samples for algal studies were also collected in the same pattern from all the fields.

Overall, 120 composite soil samples (40 $\times 3$ soil regions) were collected from the three different areas of Kuttanadu for both the soil and algal studies. Algae were identified according to the keys of Desikachary, 1959 [24] and the classification of Guiry and Guiry, 2012 [25]. The morphological parameters such as nature of the filaments, the shape and size of vegetative cells, heterocysts, akinetes, width and length of intercalary cells, presence or absence of constriction at the cross wall and of the sheath; colour of the sheath; nature of trichomes and filaments; presence or absence of heterocyst; width and length of heterocyst were taken into consideration during the identification of the taxa. Statistical analyses such as ANOVA and Pearson's correlation coefficient were carried out using the SAS 9.2 (2010) version software.

Physico-chemical characteristics of the soil, such as $\mathrm{pH}$, total organic carbon (C), total Kjeldal N, plant available P, K, Ca and Mg studied in relation to crop seasons and different stages of paddy growth in the three different regions of Kuttanadu are already reported [23].

\section{Results}

Altogether 64 species of blue-greens belonging to 22 genera and 6 orders have been found out from Kuttanadu paddy fields during the study period (2009-11). Overall distribution of them in the three soil regions in different crop-seasons and growth stages of paddy are given in Table 1. The genus Oscillatoria with 12 species dominated the soils, followed by Anabaena (9 species) and Notoc (7 species). Phormidium and Leptolyngbya with 5 species each were also found out. Out of the sixty four species, thirty species were observed in culture only, thirty three were found out from fresh soil samples and Leptolyngbya cf. battersi was the only species appeared in both culture and fresh soil samples. Relative abundance of all species is given in Figure 2. The six orders of algae observed were Chroococcales (5 genera, 9 species; 14\%), Synechocystales (3 genera, 4 species; 6\%), Nostocales (4 genera, 18 species; 28\%), Stigonematales (3 genera, 4 species; 6\%), Pseudoanabenales (1 genus and 5 species; 8\%) and Oscillatoriales (5 genera, 24 species; 38\%). Population characteristics of all species are given in Table 1. Species richness and diversity index of all groups are given in Table 2 and soil chemical characteristics in Table 3. Plates 1-4 show photographs of all the 64 species found out from Kuttanadu.

\subsection{Number of Species and Total Isolates}

The highest number of species (45 species) and total isolates (287) were observed in the Lower Kuttanadu fields during the Virippu season in the panicle crop-growth-stage. The lowest number of species (16) was found in the two crop-growth-stages of puncha season in Upper Kuttanadu; the lowest total number of isolates (76) was also observed in the Upper Kuttanadu during the puncha season at the seedling stage (Table 2). Chroococcus turgidus was the most abundant species both in the upper and Lower Kuttanadu regions. Gleothecerupestris was the most abundant one in Kayal lands. The most abundant blue-green-algae observed in Puncha season was Chroococcusturgidus, whereas the same observed in the Virippu season were Nostoccarneum and Anabaena spherica. Seven species were found specific to upper Kuttanadu soils, ten species to Lower Kuttanadu soils, whereas no cyanobacterium was found specific to Kayal lands.

\subsection{Ecological Characteristics}

The ecological characteristics studied were relative abundance, species richness, species evenness and diversity 
Table 1. Distribution and relative abundance (\%) of blue green algae of three different soil regions of Kuttanadu in relation to crop-seasons and paddy growth stages.

\begin{tabular}{|c|c|c|c|c|c|c|c|c|c|c|c|c|c|c|}
\hline \multirow{3}{*}{ No } & \multirow{3}{*}{ Cyanaophyceae sp. } & \multicolumn{4}{|c|}{$\mathrm{R} 1$} & \multicolumn{4}{|c|}{$\mathrm{R} 2$} & \multicolumn{4}{|c|}{ R3 } & \multirow{3}{*}{ Abund \% } \\
\hline & & \multicolumn{2}{|c|}{ S1 } & \multicolumn{2}{|c|}{ S2 } & \multicolumn{2}{|c|}{$\mathrm{S} 1$} & \multicolumn{2}{|c|}{ S2 } & \multicolumn{2}{|c|}{$\mathrm{S} 1$} & \multicolumn{2}{|c|}{ S2 } & \\
\hline & & G1 & G2 & G1 & G2 & G1 & G2 & G1 & G2 & G1 & G2 & G1 & G2 & \\
\hline \multicolumn{15}{|c|}{ Order: Chroococcales } \\
\hline 1 & Microcystis aeruginosa $^{* *}$ & 5 & - & 7 & - & - & - & - & 8 & 4 & 9 & - & - & 27.50 \\
\hline 2 & Gleocapsa punctata* & - & - & 9 & 10 & 6 & 8 & - & - & 5 & 6 & 9 & 10 & 52.50 \\
\hline 3 & Gleothece rupestris ${ }^{*}$ & 7 & 9 & - & - & - & - & - & - & 7 & 10 & 6 & 9 & 40.00 \\
\hline 4 & Aphanothece conferta* & 6 & 7 & 4 & 9 & - & - & - & - & - & - & - & - & 21.67 \\
\hline 5 & Chroococcus turgidus* & 5 & 8 & 8 & 9 & 8 & 8 & 7 & 9 & 8 & 4 & - & - & 61.67 \\
\hline 6 & C. limneticus ${ }^{* *}$ & - & 6 & - & - & 5 & 7 & - & - & 6 & - & - & - & 20.00 \\
\hline 7 & Chroococcus minutes $^{* *}$ & 7 & 7 & - & - & - & 7 & 8 & 10 & 7 & 6 & - & - & 43.33 \\
\hline 8 & Chroococcus minor ${ }^{*}$ & 6 & - & - & 7 & 5 & - & 6 & 8 & 5 & 8 & 6 & - & 42.50 \\
\hline 9 & Spirulina laxissima $^{*}$ & 4 & 3 & - & - & - & - & - & - & - & - & - & - & 5.83 \\
\hline
\end{tabular}

\section{Order: Synechocystales}

$\begin{array}{lcccc}10 & \text { Synechocystis aquatilis }^{* *} & - & - & - \\ 11 & \text { Aphanocapsa crassa }^{*} & 5 & 8 & 6 \\ 12 & \text { Merismopedia punctata }^{*} & 4 & 8 & 4 \\ 13 & \text { Merismopedia glauca }^{*} & 5 & 7 & -\end{array}$

\section{Order: Nostocales}

\begin{tabular}{|c|c|c|c|c|c|c|c|c|c|c|c|c|c|c|}
\hline 14 & Nostoc punctiforme ${ }^{* *}$ & - & - & - & - & 6 & 9 & 4 & - & - & 3 & 5 & - & 22.50 \\
\hline 15 & Nostoc padulosum ${ }^{*}$ & - & 6 & 6 & - & - & - & 7 & 8 & - & - & 8 & 9 & 36.67 \\
\hline 16 & Nostoc linckia ${ }^{*}$ & 5 & - & 5 & 7 & - & 4 & - & 9 & 4 & - & 7 & 9 & 41.67 \\
\hline 17 & Nostoc spongiaeforme ${ }^{*}$ & 3 & 4 & 2 & 3 & - & - & - & - & - & - & - & - & 10.00 \\
\hline 18 & Nostoc carneum ${ }^{*}$ & 2 & - & 6 & 9 & 4 & - & 8 & 10 & 3 & - & 8 & - & 41.67 \\
\hline 19 & Nostoc ellipsosporum $^{*}$ & - & 4 & 5 & 8 & 5 & 7 & 9 & 9 & - & 7 & 9 & - & 52.50 \\
\hline 20 & Nostoc commune ${ }^{*}$ & 6 & - & 4 & 8 & 5 & 6 & 8 & 10 & - & - & - & 7 & 45.00 \\
\hline 21 & Anabaena sphaerica $^{* *}$ & - & - & 5 & 7 & - & - & 7 & 9 & 4 & 6 & 6 & 7 & 42.50 \\
\hline 22 & Anabaena oryzae $e^{* *}$ & - & - & 4 & 5 & - & - & 2 & 4 & 3 & - & 4 & 6 & 23.33 \\
\hline 23 & Anabaena fertilissima* & - & - & 5 & 6 & - & - & 5 & 5 & 4 & 5 & 5 & 5 & 33.33 \\
\hline 24 & Anabaena gelatinicola* & - & - & 1 & 2 & - & - & 1 & 2 & 2 & - & 2 & 2 & 10.00 \\
\hline 25 & Anabaena orientalis $^{* * *}$ & - & - & 2 & 3 & - & - & 1 & 1 & 1 & 1 & 2 & 3 & 11.67 \\
\hline 26 & Anabaena variabilis ${ }^{*}$ & - & - & 2 & 2 & - & - & 2 & 2 & - & 2 & 3 & 3 & 13.33 \\
\hline 27 & Anabaena torulosa* & - & - & 5 & 7 & - & - & 4 & 6 & 4 & 7 & 5 & - & 31.67 \\
\hline 28 & A. oscillatorides ${ }^{* *}$ & - & - & 2 & 2 & - & - & 5 & 7 & 5 & 6 & 6 & - & 27.50 \\
\hline 29 & Anabaena constricta $^{* *}$ & - & - & 2 & 2 & - & - & 2 & 3 & 4 & 4 & 6 & - & 19.17 \\
\hline 30 & Nodularia spumigena ${ }^{*}$ & - & - & - & - & - & 7 & - & 9 & - & - & - & - & 13.33 \\
\hline 31 & Aulosira prolifica ${ }^{*}$ & - & - & 6 & 7 & - & 5 & - & 5 & - & - & - & - & 19.17 \\
\hline
\end{tabular}

\section{Order: Stigonematales}




\section{Continued}

\begin{tabular}{|c|c|c|c|c|c|c|c|c|c|c|c|c|c|c|}
\hline 33 & H. flagelliformae ${ }^{*}$ & - & 9 & 8 & 8 & - & - & - & - & 10 & 10 & 9 & - & 45.00 \\
\hline 34 & Westiellopsis prolifica ${ }^{*}$ & - & - & - & - & 9 & 10 & - & - & - & - & - & - & 15.83 \\
\hline 35 & Scytonema stuposum ${ }^{* *}$ & - & - & - & - & - & - & 4 & 5 & - & 4 & - & 4 & 14.17 \\
\hline \multicolumn{15}{|c|}{ Order: Pseudoanabenales } \\
\hline 36 & Leptolyngbya boryana ${ }^{*}$ & - & - & 6 & 4 & 4 & 2 & 8 & 9 & - & - & - & - & 27.50 \\
\hline 37 & Leptolyngbya tenui** $^{* *}$ & - & - & - & 7 & 7 & 5 & - & 10 & - & - & - & - & 24.17 \\
\hline 38 & L. cf. battersi $i^{* * *}$ & - & - & 9 & - & 7 & - & 8 & 8 & - & - & - & - & 26.67 \\
\hline 39 & L. circumcreta* & - & - & 3 & 5 & 1 & 3 & 4 & 5 & - & - & - & - & 17.50 \\
\hline 40 & Leptolyngbya $s p^{*}$ & - & - & 2 & 2 & 2 & 2 & 5 & 7 & - & - & - & - & 16.67 \\
\hline \multicolumn{15}{|c|}{ Order: Oscillatoriales } \\
\hline 41 & Arthrospira jenneri $^{* *}$ & 2 & 3 & - & - & - & - & - & - & - & - & - & - & 4.17 \\
\hline 42 & Oscillatoria margaritifera $^{* *}$ & - & - & - & - & - & 7 & 8 & 8 & - & - & - & - & 19.17 \\
\hline 43 & Oscillatoria sancta ${ }^{* *}$ & - & - & - & - & 8 & - & - & 9 & - & - & - & 10 & 22.50 \\
\hline 44 & Oscillatoria limosa** & - & - & - & - & - & - & 10 & 10 & - & - & 9 & 9 & 31.67 \\
\hline 45 & Oscillatoria curviceps ${ }^{* * *}$ & - & - & - & - & - & - & 7 & 7 & - & - & 8 & 9 & 25.83 \\
\hline 46 & Oscillatoria princeps ${ }^{* *}$ & - & - & - & - & 6 & 7 & 9 & 8 & 5 & 5 & 10 & 10 & 50.00 \\
\hline 47 & Oscillatoria amphibia* & 4 & 4 & - & - & - & - & - & - & - & - & - & - & 6.67 \\
\hline 48 & Oscillatoria tenuis ${ }^{* *}$ & - & - & - & - & 3 & 2 & 7 & 7 & - & - & 6 & 8 & 27.50 \\
\hline 49 & Oscillatoria proteus $^{* *}$ & - & - & - & - & - & - & 1 & 2 & - & - & 2 & 1 & 5.00 \\
\hline 50 & O. simplicissima ${ }^{* *}$ & - & - & - & - & - & - & 2 & 2 & - & - & 3 & 5 & 10.00 \\
\hline 51 & Oscillatoria irrigua $^{* *}$ & - & - & - & - & - & - & 1 & 1 & - & - & 1 & 1 & 3.33 \\
\hline 52 & Oscillatoria amoena ${ }^{* *}$ & - & - & - & - & - & - & 1 & - & - & - & 1 & 2 & 3.33 \\
\hline 53 & Oscillatoria rubescens ${ }^{* *}$ & - & - & - & - & - & 5 & 4 & - & - & 3 & 4 & 5 & 17.50 \\
\hline 54 & Phormidium tenue ${ }^{* *}$ & - & - & - & - & 1 & 1 & 1 & 1 & - & - & 1 & 1 & 5.00 \\
\hline 55 & P. purpurascens ${ }^{* *}$ & - & - & - & - & - & - & 7 & 9 & - & - & - & - & 13.33 \\
\hline 56 & P. ambiguum ${ }^{* *}$ & - & - & - & - & - & 3 & 3 & 5 & - & - & - & - & 9.17 \\
\hline 57 & Phormidiumin undatum $^{* *}$ & - & - & - & - & - & - & - & - & - & - & 2 & 4 & 5.00 \\
\hline 58 & P. papyraceum ${ }^{* *}$ & - & - & - & - & - & 1 & - & 3 & 1 & - & - & - & 4.17 \\
\hline 59 & Lyngbya dendrobia ${ }^{* *}$ & - & - & - & - & - & - & 9 & 8 & 9 & - & - & - & 21.67 \\
\hline 60 & Lyngbya ceylanica $^{* *}$ & - & - & - & - & 4 & - & 3 & 7 & - & - & 4 & 4 & 18.33 \\
\hline 61 & Lyngbya confervoides $^{* *}$ & - & - & - & - & - & - & - & - & 1 & 1 & 1 & 2 & 4.17 \\
\hline 62 & Lyngbya martensiana $^{* *}$ & - & - & - & - & - & - & 2 & 3 & - & - & - & - & 4.17 \\
\hline 63 & Microcoleus vaginatus ${ }^{*}$ & - & - & - & - & - & - & 7 & 7 & - & - & - & 8 & 18.33 \\
\hline 64 & M. accutissimus ${ }^{*}$ & - & - & - & - & 5 & - & 8 & 6 & - & - & 7 & 7 & 27.50 \\
\hline & Total species/genera & $16 / 9$ & $16 / 10$ & $28 / 11$ & $27 / 9$ & $20 / 9$ & $22 / 8$ & $41 / 10$ & $45 / 13$ & $23 / 10$ & $21 / 9$ & $32 / 10$ & $28 / 8$ & \\
\hline
\end{tabular}

Species found only in culture ${ }^{*}$, Species found in fresh soil sample ${ }^{* *}$, Species found both in fresh soil sample and in the culture**. 
Table 2. Ecological Characteristics of blue green algae in the Kuttanadu paddy soils.

\begin{tabular}{|c|c|c|c|c|c|c|c|}
\hline Region & Season & Growth Stage & No. of Species & Total Isolates & Sps rich & Div. Ind & Sps. Evn \\
\hline \multirow{4}{*}{ U.K } & \multirow{2}{*}{ S1 } & G1 & 16 & 76 & 0.383 & 1.38 & 0.498 \\
\hline & & $\mathrm{G} 2$ & 16 & 101 & 0.383 & 1.38 & 0.498 \\
\hline & \multirow{2}{*}{ S2 } & G1 & 28 & 135 & 0.671 & 1.68 & 0.504 \\
\hline & & G2 & 27 & 164 & 0.647 & 1.66 & 0.504 \\
\hline \multirow{4}{*}{ L.K } & \multirow{2}{*}{ S1 } & G1 & 20 & 101 & 0.479 & 1.5 & 0.501 \\
\hline & & G2 & 22 & 116 & 0.527 & 1.55 & 0.502 \\
\hline & \multirow{2}{*}{$\mathrm{S} 2$} & G1 & 41 & 210 & 0.983 & 1.88 & 0.507 \\
\hline & & G2 & 45 & 287 & 1.078 & 1.93 & 0.508 \\
\hline \multirow{4}{*}{ K.L } & \multirow{2}{*}{ S1 } & G1 & 23 & 110 & 0.551 & 1.58 & 0.502 \\
\hline & & G2 & 21 & 116 & 0.503 & 1.53 & 0.501 \\
\hline & \multirow{2}{*}{ S2 } & G1 & 32 & 165 & 0.767 & 1.75 & 0.505 \\
\hline & & G2 & 28 & 160 & 0.671 & 1.68 & 0.504 \\
\hline
\end{tabular}

Table 3. Average Soil Chemical characteristics of wetland paddy fields of Kuttanadu (Ray et al., 2014).

\begin{tabular}{|c|c|c|c|c|c|c|c|c|c|c|}
\hline \multirow{2}{*}{ No } & \multirow{2}{*}{ Location } & \multirow{2}{*}{$\begin{array}{l}\text { Crop } \\
\text { season }\end{array}$} & \multirow{2}{*}{$\begin{array}{l}\text { Growth } \\
\text { stage }\end{array}$} & \multirow{2}{*}{ Average $\mathrm{p}^{\mathrm{H}}$} & \multirow{2}{*}{$\begin{array}{l}\text { Average } \\
\text { TN (\%) }\end{array}$} & \multirow{2}{*}{$\begin{array}{l}\text { Average } \\
\text { OC (\%) }\end{array}$} & \multicolumn{4}{|c|}{ Average available nutrients (kg/ha) } \\
\hline & & & & & & & $\mathrm{P}$ & $\mathrm{K}$ & $\mathrm{Ca}$ & $\mathrm{Mg}$ \\
\hline 1 & U K & Puncha & Seedling & 3.94 & 0.429 & 3.07 & 10.86 & 998.6 & 3858.8 & 788.8 \\
\hline 2 & U K & Puncha & Panicle & 3.88 & 0.392 & 4.06 & 18.26 & 656.9 & 2378.6 & 512.2 \\
\hline 3 & U K & Virippu & Seedling & 3.4 & 0.289 & 0.94 & 129 & 298.7 & 441.4 & 177.7 \\
\hline 4 & U K & Virippu & Panicle & 3.65 & 0.459 & 2.63 & 110 & 496.5 & 758.2 & 205.5 \\
\hline 5 & L K & Puncha & Seedling & 4 & 0.413 & 3.97 & 7.14 & 855 & 1842.4 & 785.8 \\
\hline 6 & L K & Puncha & Panicle & 4.29 & 0.374 & 3.54 & 25.34 & 389.2 & 2841.7 & 840.2 \\
\hline 7 & L K & Virippu & Seedling & 3.74 & 0.37 & 1.68 & 109 & 316.7 & 1078 & 321.9 \\
\hline 8 & L K & Virippu & Panicle & 3.7 & 0.52 & 2.16 & 95 & 408.9 & 1437.4 & 377.4 \\
\hline 9 & K L & Puncha & Seedling & 4.03 & 0.39 & 3.28 & 9.6 & 391.2 & 1249.6 & 748.5 \\
\hline 10 & K L & Puncha & Panicle & 4.61 & 0.435 & 3.7 & 9.46 & 396 & 2331.8 & 992.1 \\
\hline 11 & K L & Virippu & Seedling & 4.05 & 0.4 & 2.49 & 129 & 440.9 & 1466.6 & 407.3 \\
\hline 12 & K L & Virippu & Panicle & 4.23 & 0.431 & 2.68 & 58 & 408.3 & 1618.7 & 399.9 \\
\hline
\end{tabular}

\section{Orders of Cyanophyceae}

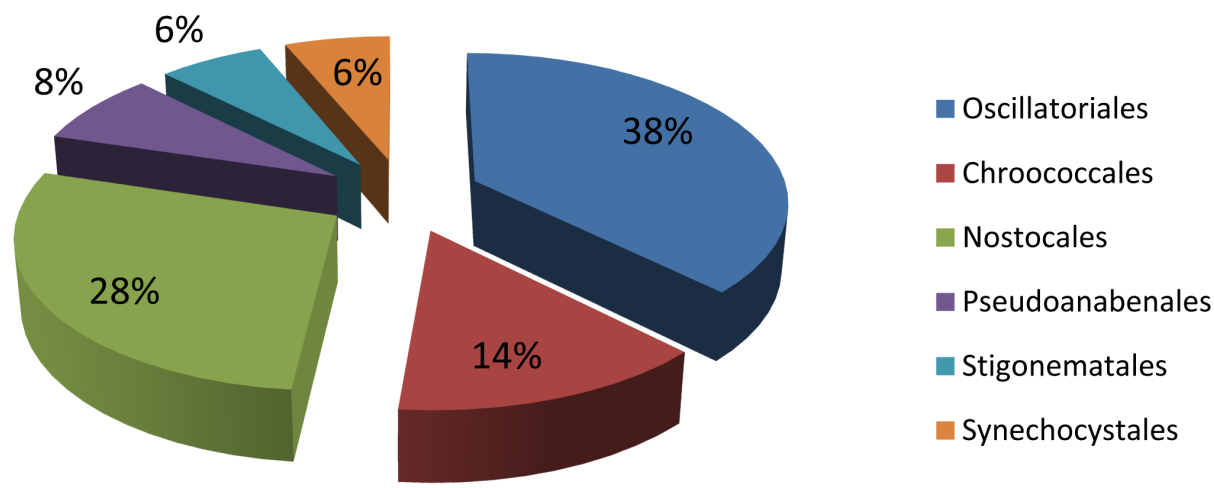

Figure 2. Diversity of bluegreen algae in Kuttanadu paddy soils. 

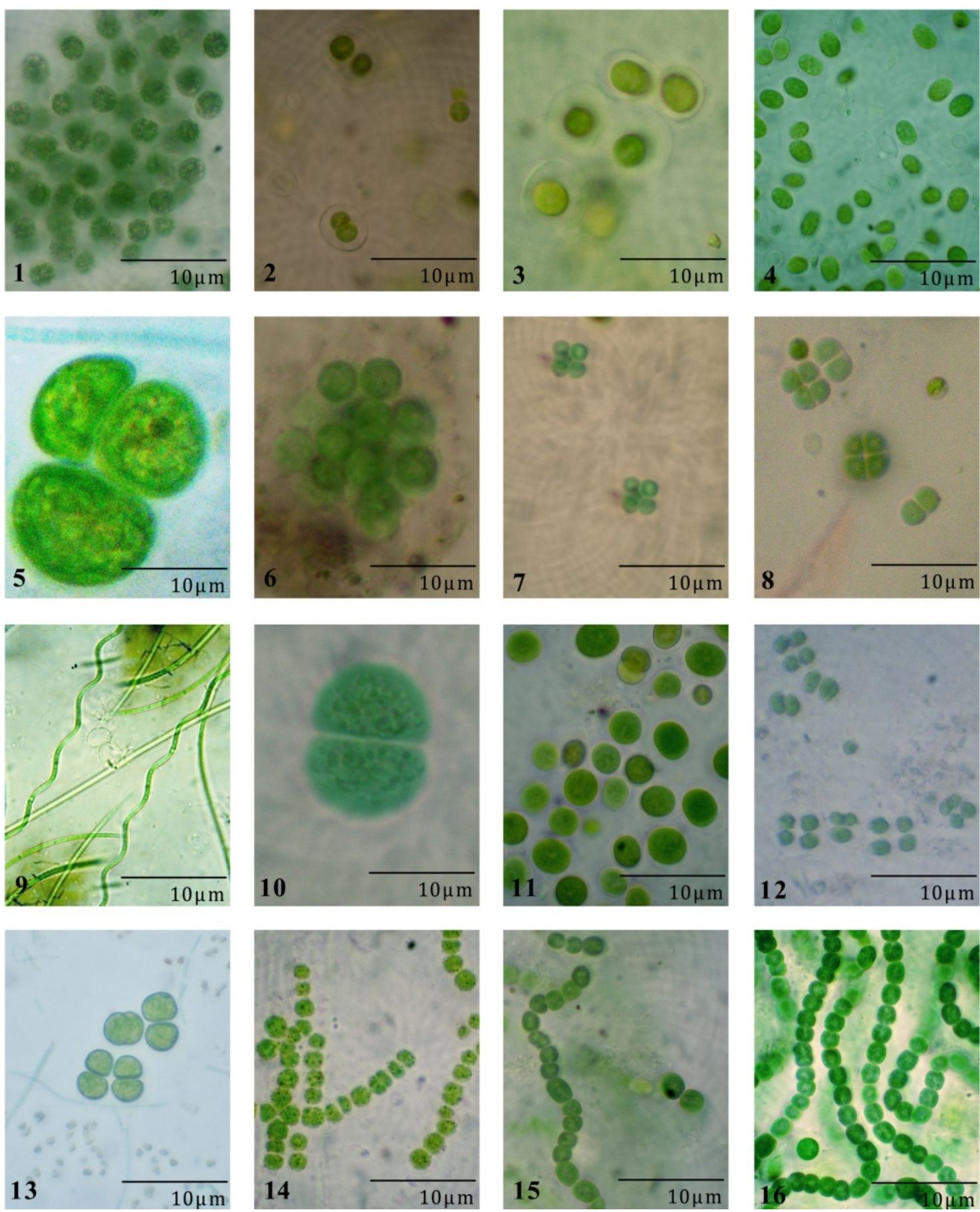

Plate 1. Figures 1-16: 1. Microcystis aeruginosa (Kutzing) Kutzing ${ }^{* *}$, 2.Gleocapsa punctata Nageli*, 3. Gleothece rupestris (Lyngb) Bornet", 4. Aphanothe ceconferta Richter ${ }^{*}$, 5. Chroococcus turgidus (Kutzing) Nageli ${ }^{*}$, 6. Chroococcus limneticus Lemmermann ${ }^{* *}$, 7. Chroococcus minutes (Kutz.) Nageli**, 8. Chroococcus minor (Kutz) Nageli", 9. Spirulina laxissima G. S. West*, 10. Synechocystis aquatilis Sauv ${ }^{* *}, 11$. Aphanocapsa crassa Ghose*, 12. Merismopedia punctata Meyen*, 13. Merismopedia glauca (Ehrenb.) Nag. , 14. Nostoc punctiforme (Kuz.) Hariot ${ }^{* *}, 15$. Nostoc padulosum Kutzing ex Born.et Flah", 16. Nostoc linckia (Roth) Bornet ex Born. Flah*.

index [26]. The relative abundance of the all sixty four green algal species was calculated from 120 soil samples representing the three different soil regions of Kuttanadu. The most dominant species in Kuttanadu was Chroococcus turgidus with relative abundance of $61.67 \%$, followed by Gleocapsa punctata (52.5\%), Nostoc ellipsorum 

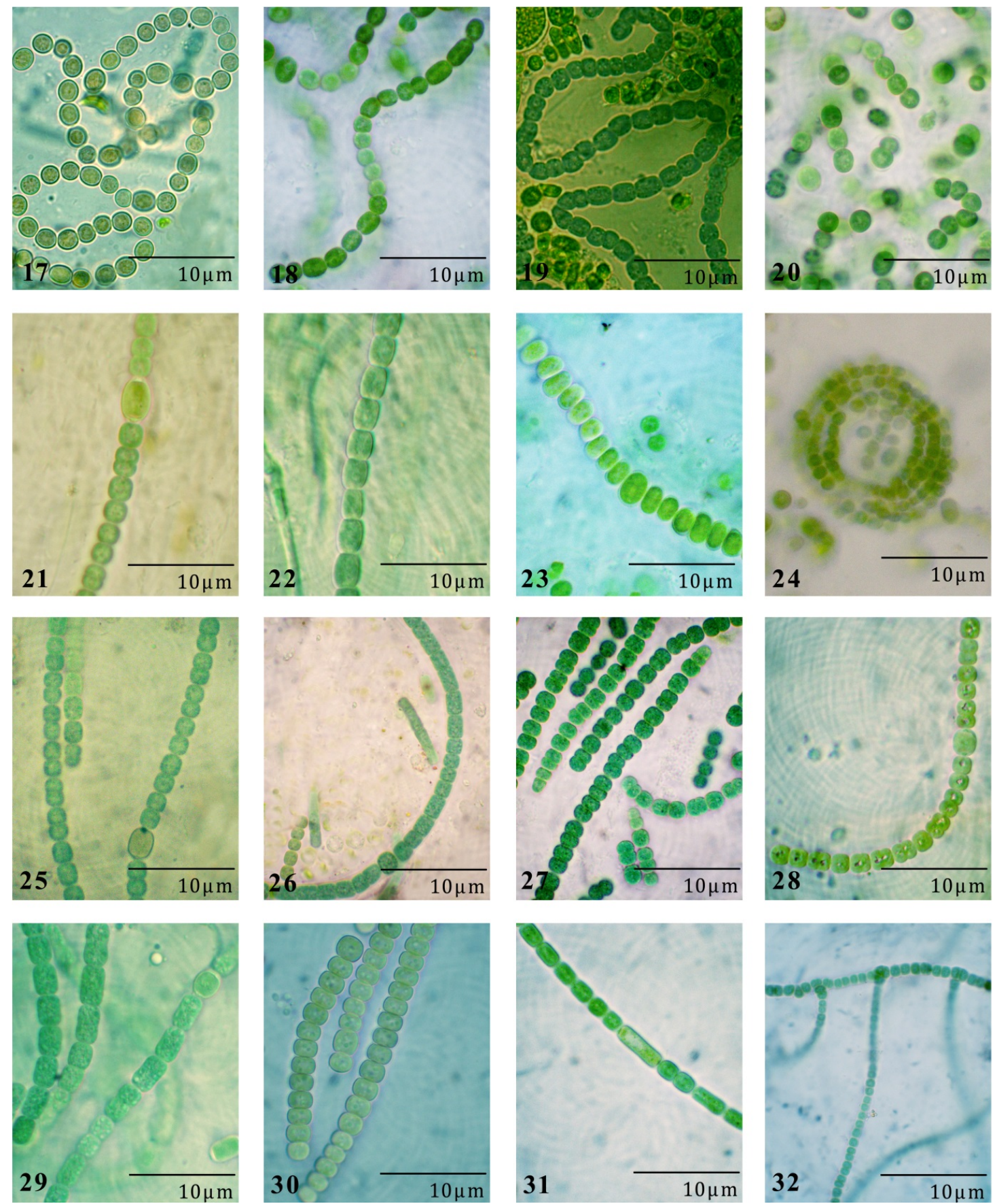

Plate 2. Figures 17-32: 17. Nostoc spongiaeforme Agardh ex Born.et Flah, 18. Nostoc carneum Ag.ex Born.et Flah", 19. Nostoc ellipsosporum (Desm.) Rabenh.ex Born.et Flah., 20. Nostoc commune Vaucher ex Born.et.

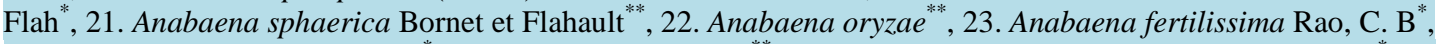
24. Anabaena gelatinicola Ghose*, 25. Anabaena orientalis ${ }^{* *}, 26$. Anabaena variabilis Kutzing ex Born. Flah*, 27. Anabaena torulosa (Carm.) Lagerh.ex Born.et Flah", 28. Anabaena oscillatorides ${ }^{* *}$, 29. Anabaena constricta ${ }^{* *}, 30$. Nodulariaspumigena Mertens ex Born.et Flah*, 31. Aulosira prolific Bharadwaja*, 32. Hapalosiphon welwitschii West \& West ${ }^{*}$.

(52.5\%) and Oscillatoria princeps (50\%). The least abundant algae were Oscillatoria irrigua and O. amoena; each with a relative abundance $3.33 \%$. Chroococcus turgidus was the most abundant species in Upper and Lower Kuttanadu with relative abundances $75 \%$ and $80 \%$ respectively. Gleothece rupestris was found to be the most 

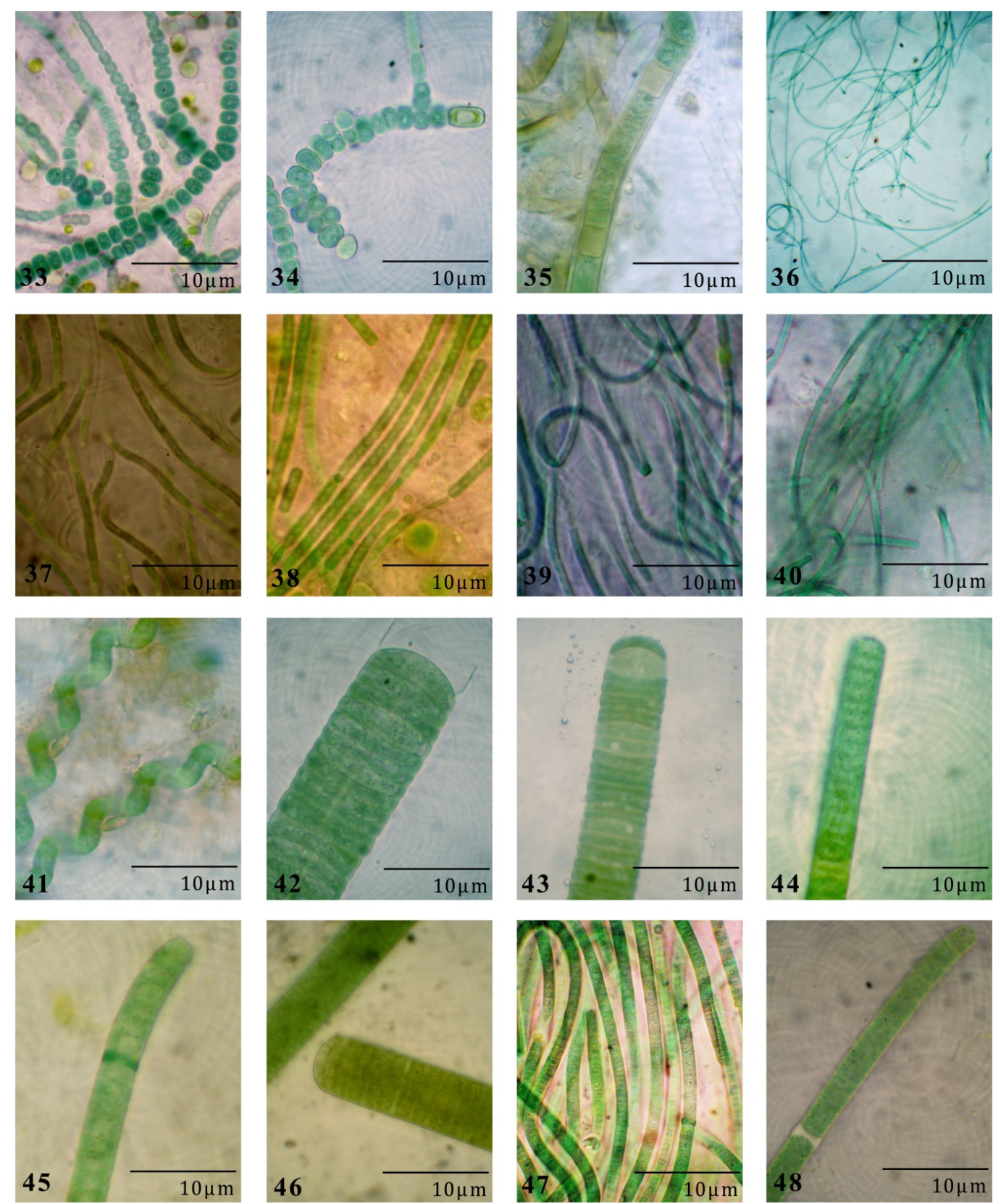

Plate 3. Figures 33-48: 33. Hapalosiphon flagelliformae (Schmidle) Forti, 34. Westiellopsis prolifica Janet, 35. Scytonema stuposum (Kutzing) Bornet ex Born.et Flah ${ }^{* *}$, 36. Leptolyngbya boryana*, 37. Leptolyngbya tenuis $^{* *}$, 38. Leptolyngbya cf. battersi***, 39. Leptolyngbya circumcreta, 40. Leptolyngbya sp ${ }^{*}$, 41. Arthrospira jenneri Stizenb.ex Gomont ${ }^{* *}$, 42. Oscillatoria margaritifera (Kutz.) Gomontt ${ }^{* *}$, 43. Oscillatoria sancta (Kutz.) Gomontt ${ }^{* *}$, 44. Oscillatoria limosa C. Agardh ${ }^{* *}$, 45. Oscillatoria curviceps Ag.ex Gomont ${ }^{* *}, 46$. Oscillatoria princepsVaucher ex Gomont ${ }^{* *}$, 47. Oscillatoria amphibian Ag.ex.Gomont, 48. Oscillatoria tenuis Ag. exGomont*.

abundant species in Kayal lands with relative abundance of 80\% (Table 1). Species richness (Sps rich) of blue-greens in Kuttanadu wetlands showed a variation from 0.383 - 1.078 along the three different soil regions during the different crop seasons and crop stages. The species richness was found maximum (1.078) in Lower Kuttanadu, during Virippu season at panicle stage. Minimum species richness 0.383 was noticed in Upper Kuttanadu during seedling stage and panicle stage of Puncha cultivation (Table 2). Diversity index (Div. Ind) of blue-greens varied from 1.38 to 1.93 in different regions of Kuttanadu during the diferent crop seasons and crop 

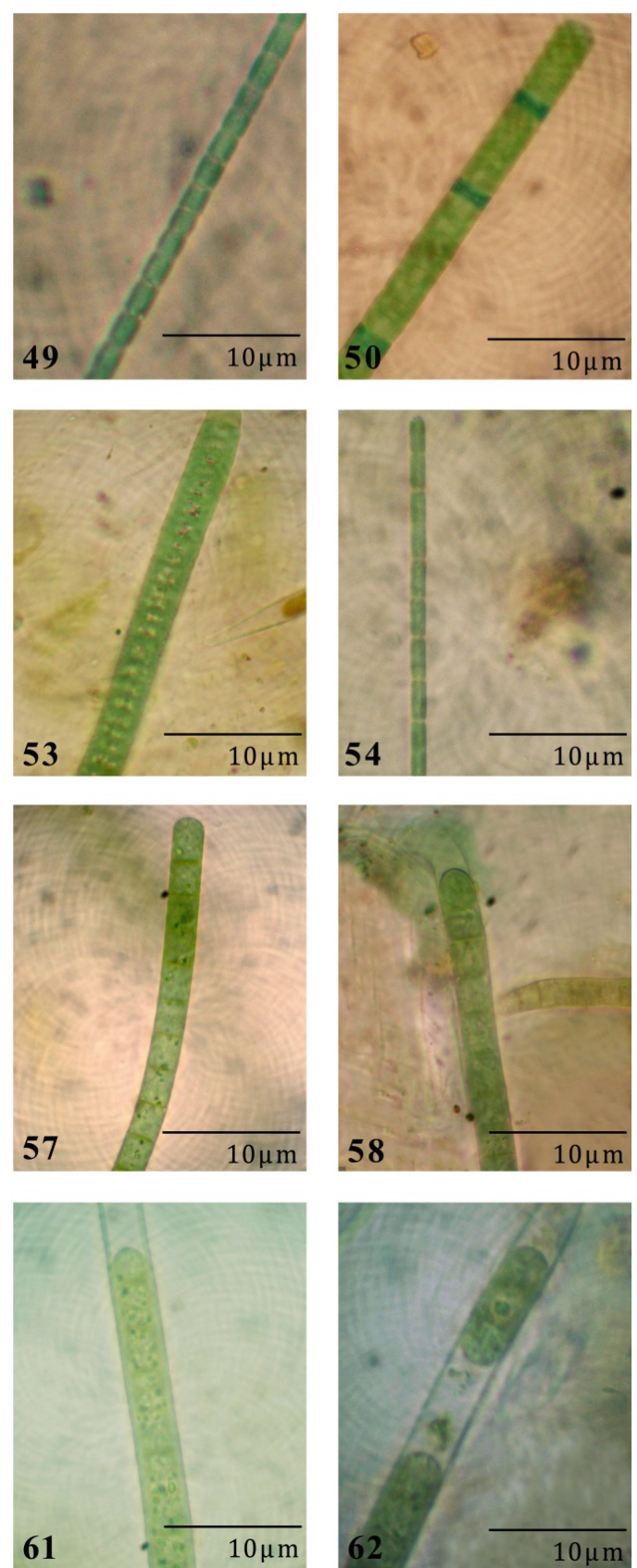
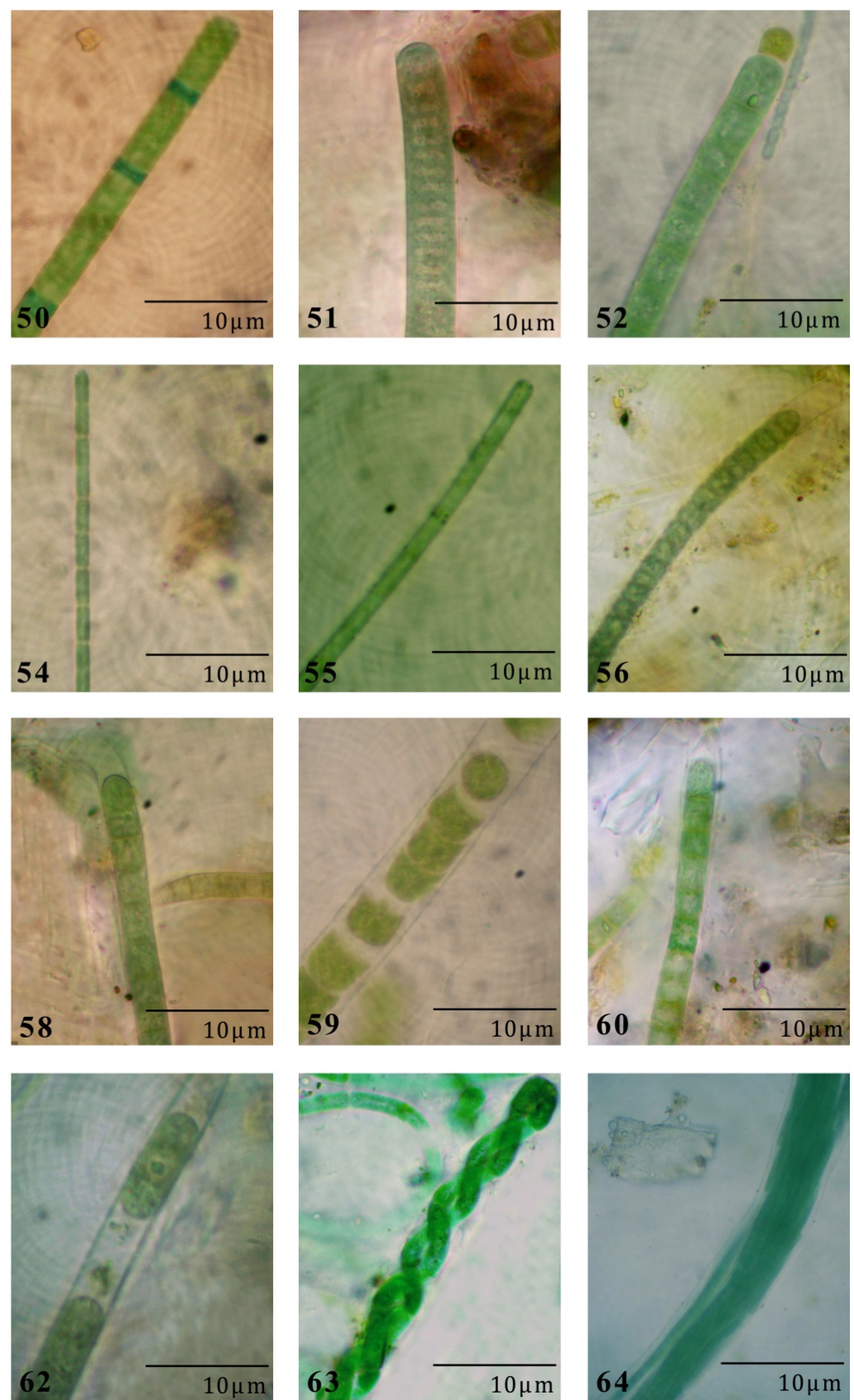

Plate 4. Figures 49-64: 49. Oscillatoria proteusSkuja**, 50. Oscillatoria simplicissima Gomont ${ }^{* *} 51$. Oscillatoria irrigua (Kutz.) Gomont ${ }^{* *}$, 52. Oscillatoria amoena (Kutz.) Gomont ${ }^{* *}$, 53. Oscillatoria rubescens DC ex Gomont ${ }^{* *}$, 54. Phormidium tenue (Menegh.) Gomont ${ }^{* *}$, 55. Phormidium purpurascens (Kutz.) Gomont $^{* *}$, 56. Phormidium ambiguum Rao, C. B. ${ }^{* *}$, 57. Phormidium inundatum Kutzing ex Gomont ${ }^{* *}, 58$. Phormidium papyraceum Kutzing ex Gomont**, 59. Lyngbya dendrobia Bruhl et Biswas**, 60. Lyngbya ceylanica Wille**, 61. Lyngbya confervoides C. Ag.exGomont**, 62. Lyngbya martensiana Menegh. exGomont ${ }^{* *}$, 63. Microcoleus vaginatus (Vaucher) Gomont", 64. Microcoleus accutissimus Gardner*.

stages. The diversity index was found maximum in Lower Kuttanadu, during Virippu season at both the seeding and panicle stage. Minimum diversity index was noticed in upper Kuttanadu during Puncha season at the seedling stage (Table 2). Species evenness (Sps. Evn) varied from 0.498 to 0.508 in different soil regions of Kuttanadu during the different crop-seasons and crop-growth-stages. The species evenness was found maximum in lower Kuttanadu, during Virippu season at the panicle stage. Minimum species evenness was noticed in upper 
Kuttanadu during the seedling and panicle stage of Puncha cultivation (Table 2).

\subsection{Environmental Correlations}

Correlations of species richness, diversity index, number of species and total isolates to soil regions, crop seasons and crop-growth-stages of paddy and to soil parameters such as $\mathrm{pH}, \mathrm{N}, \mathrm{P}, \mathrm{K}, \mathrm{Ca}$ and $\mathrm{Mg}$ using were assessed using Pearson's correlation coefficient. The species richness and diversity index of blue-greens showed significant negative correlation to organic carbon $(\mathrm{P}<0.000)$, phosphorus, potassium $(\mathrm{P}<0.000)$, calcium $(\mathrm{P}<$ $0.000)$, and magnesium $(\mathrm{P}<0.000)$. The species richness and diversity index showed positive correlation $(\mathrm{P}<$ 0.000 ) to crop seasons. Soil regions and crop-growth-stages had no correlation to species richness and diversity index. Total number of isolates and number of species present were positively correlated $(\mathrm{P}<0.000)$ to crop seasons. The number of blue-greens were found negatively correlated to $\mathrm{pH}(\mathrm{P}<0.000)$, Organic Carbon $(\mathrm{P}<$ $0.000)$, Potassium $(\mathrm{P}<0.000)$, Calcium $(\mathrm{P}<0.000)$ and Magnesium $(\mathrm{P}<0.000)$. A positive correlation was found between Phosphorus $(\mathrm{P}<0.000)$ and number of species observed. Total number of blue green algal isolates were positively correlated with Total Nitrogen $(\mathrm{P}<0.005)$, and total Phosphorus $(\mathrm{P}<0.000)$. Negative correlation existed between Organic Carbon $(\mathrm{P}<0.000)$, Potassium $(\mathrm{P}<0.002)$, Calcium $(\mathrm{P}<0.001)$ and Magnesium $(\mathrm{P}<0.000)$.

\section{Discussion}

High species diversity of any particular group of organisms in a natural habitat can be interpreted as the essentiality of the same in the system as a major biotic component. Paddy fields are unique wet soil habitats [27], very suitable for blue-green-algae to flourish [12]. In the paddy fields their significant contributions to soil fertility in terms of the physico-chemical, biological and soil-water relations [6] are well known. Roles of cyanobacteria in soil conditioning [28] and as soil bio-indicators [29] are also important. High diversity of cyanobacteria (64 species) in Kuttanadu paddy fields reiterates these facts.

In the Kuttanadu fields, among the total species 38\% are from the order Oscillatoriales. Oscillatoria and Phormi-dium are the common tolerant genera of polluted areas [30] [Therefore, the prevalence of Oscillatoriales as the dominant group in Kuttanadu, reveals the current ecological status of the last three decades of green revolution farming in Kuttanadu agricultural soils. Nostoc is another genus reported to be common in paddy fields [7]. In these fields also the genus is found common with about 7 species.

Lower Kuttanadu with the highest values for species number (47), total isolates, species richness and species evenness have unique soil characteristics. The clayey loam texture with comparatively high amount of decomposing organic matter and nitrogen $(0.37 \%$ to $0.52 \%)$ may be the reason for highest algal community parameters noted here. Role of soil texture in Cyanobacterial variations, growth and distribution [31] and also the significance of physico-chemical properties of soils to blue greens [32] are well known. Moreover, the clayey soil in the region with significantly higher amount of calcium might be another reason for the presence of higher growth of blue-greens in this soil region than in other soil regions.

In terms of soil pH the upper Kuttanadu soil was found to be more acidic (3.4 to 3.9) than those of Lower Kuttanadu (3.7 to 4.2) and Kayal lands (4 to 4.6). Presence of the lowest values of species number (16) and total isolates (76) in the upper Kuttanadu soils may be attributed to this fact as well. In general acidic soils are considered as are stressed environments for Cyanophytes [6] [9] [33]. The negative correlation observed between soil $\mathrm{pH}$ and Cyanobacterial population in Kuttanadu paddy fields is in agreement with such previous observations. However, report of [20] large number of species of cyanobacteria in extreme acidic environments also exists. Similarly report [34] of plenty of Cyanobacterial population in soils of highly alkaline $\mathrm{pH}$ is also available. However, the exact relationship of $\mathrm{pH}$ to the growth of specific Cyanobacterial species in soils is yet to be confirmed.

The present study revealed that highest number of species and total isolates were observed in the panicle cropgrowth-stage during the monsoon season. Water availability is a primary factor in determining the abundance of algae [35] [36] in soils. In general, Cyanobacteria are observed in plenty during wet seasons and less in dry environment [34]. However, certain authors have reported the occurrence of maximum Cyanophyceae during winter [37]. Light intensity is an important factor that contributes to the algal floral composition in natural environments [14]. In the present study, a gradual increase of Cyanobacterial population was observed along with the advancement in paddy growth stages. This is in agreement with previous findings [38]. But in general, in paddy 
fields, the early part of cultivation cycle show more algal growth [27] [33] [39]. Increasing canopy of the crops causes a decrease in light intensity reaching the soil surface and there will be depletion of the nutrients, particularly nitrogen as the rice advances in its growth stage. However, report of decreased light intensity and nitrogen level along with the growth of the crops in fields favoring excessive growth of blue-greens also exists [12]. It is also known that the blue-greens, in general are sensitive to high light intensities [5].

Seasonal variations are main factors that have major roles on microalgal growth and diversity [40] in soils. Cyanobacterial populations are known to exhibit wide seasonal variations in both colony size and prevalence of heterocysts [41]. In Kuttanadu fields, total number of isolates, number of species present, species richness and diversity index were found positively correlated to crop seasons.

The concentration and quality of nutrients are probably more important in the blue-green-algal diversity. Availability of phosphates and nitrates are important factors that favor the abundance of cyanophyceae in wetlands [29]. Actually, the N:P ratio has influence on cyanobacterial abundance According to [42]. In the present study also total number of blue-green-algal isolates was found positively correlated to the amount of total $\mathrm{N}$ and $\mathrm{P}$ in soils. Phosphorus application has immediate effect on the growth blue-greens in soils [43]. In the current investigation, the total number of blue green algal isolates showed negative correlation with organic carbon, Calcium and Magnesium. This is in conformity with the report that low carbon favors richness of cyanophyta in soils [44]. However, calcium and phosphorus have decisive effects on algal abundance in soils [43].

High amount of ecological information on blue-greens in their natural surroundings is essential to the development of correct applications of indigenous blue-greens as bio-fertilizers in paddy fields [27]. Creation of such a data base require information on ecology of blue-greens from as many diverse fields as possible from different corners of the world. Bio-fertilizers are essential to avoid harmful effects of excessive applications of chemical fertilizers. In order to develop procedures of correct applications of blue-greens as bio-fertilizers at successive stages of paddy cultivation, information on the natural community of Cyanophytes from diverse paddy environments is essential. Apart from the simple analysis, documentation and assessment of cyanobacteria from paddy fields, their correlations to nutrient status of paddy fields is also highly relevant ecological information. Since chemical fertilizer applications inhibit natural nitrogen fixation by algae [45] correlations of natural community of Cyanophytes to NPK in soils is highly essential. Therefore, the present kind of investigation on the diversity of blue-greens in cultivated paddy fields is highly relevant. It also offered the possibility of finding out tolerant species of bio-fertilizer grade blue-greens from paddy fields. Knowledge of such species may enable us to find out tolerant groups of blue-greens, which may be able to act along with moderate amount of chemical fertilizers in the paddy fields, essential to optimum yields in integrated approaches.

\section{Conclusion}

The present investigation has revealed that Kuttanadu wetland paddy soils are very rich in blue green algal diversity, dominated mostly by species from the order Oscillatoriales (38\%). Certain specific species unique to the soil regions, seasons and growth stages in Kuttanadu are also revealed. Species richness and the number of species were found positively correlated to crop seasons. The highest number of isolates (287) and the highest number of species (45) were observed in the Lower Kuttanadu soil region, during the Virippu season, at panicle growth stage of the crop. Algal biodiversity appeared to be the maximum during monsoon season throughout the region. Species richness was the highest in Lower Kuttanadu soils and the lowest in Kayal lands. Chroococcus turgidus was the most abundant blue green algae in Kuttanadu, in both Upper Kuttanadu and Lower Kuttanadu soils. In Kayal lands the same was Gleotheca rupestris.

\section{References}

[1] Ghadai, A.K., Sahoo, S. and Raut, S. (2010) Agroecological Survey of Cyanobacterial Population in Paddy Field Soils of Gunupur. International Journal of Agricultural Sciences, 2, 28-32. http://dx.doi.org/10.9735/0975-3710.2.2.28-32

[2] Choudhary, A.T.M.A. and Kennedy, I.R. (2004) Prospects and Potentials for System of Biological Nitrogen Fixation in Sustainable Rice Production. Biology and Fertility of Soils, 39, 219-227. http://dx.doi.org/10.1007/s00374-003-0706-2

[3] Reynaud, P.A. and Roger, P.A. (1978) Nitrogen Fixing Algal Biomass in Senegal Rice Fields. Ecological Bulletins (Stockholm), 26, 148-157.

[4] Pereira, I., Moya, M., Reyes, G. and Kramm, V. (2005) A Survey of Heterocystous Nitrogen Fixing Cyanobacteria in Chilean Rice Fields. Gayana Botanica, 62, 26-32. http://dx.doi.org/10.4067/S0717-66432005000100005 
[5] Song, T., Martensson, L., Eriksson, T., Zheng, W.W. and Rasmussen, U. (2005) Biodiversity and Seasonal Variation of the Cyanobacterial Assemblage in a Rice Paddy Field in Fujian, China. FEMS Microbiology Ecology, 54, 131-140. http://dx.doi.org/10.1016/j.femsec.2005.03.008

[6] Nayak, S. and Prasanna, R. (2007) Soil pH and Its Role in Cyanobacterial Abundance and Diversity in Rice Field Soils. Applied Ecology and Environmental Research, 5, 103-113. http://dx.doi.org/10.15666/aeer/0502 103113

[7] Begum, T.Z.N., Mandal, R. and Amin, F.B. ( 2008) Quantification and Nitrogen Fixation of Cyanobacteria in Rice Field Soils of Bangladesh. Bangladesh Journal of Botany, 37, 183-188. http://dx.doi.org/10.3329/bjb.v37i2.1728

[8] Choudhary, K.K. (2009) Occurence of Chroococcaceae during Rice Cultivation in North Bihar, India. Bangladesh Journal of Plant Taxonomy, 16, 57-63

[9] Selvi, T.K. and Sivakumar, K. (2011) Cynobacterial Diversity and Related Physico-Chemical Parameters in Paddy Fields of Cuddalore District, Tamil Nadu. International Journal of Research in Environmental Science and Technology, 1, 7-15

[10] de Mulé, M.C.Z., de Caire, G.Z., de Cano, M.S., Palma, R.M. and Colombo, K. (1999) Effect of Cyanobacterial Inoculation and Fertilizers on Rice Seedlings and Post Harvest Soil Structure. Communications in Soil Science and Plant Analysis, 30, 97-107. http://dx.doi.org/10.1080/00103629909370187

[11] Shubert, L.E. and Starks, T.L. (1980) Soil Algal Relationships from Surface Mined Soils. British Phycological Journal, 15, 417-428. http://dx.doi.org/10.1080/00071618000650421

[12] Roger, P.A. and Reynaud, P.A. (1982) Free Living Blue Green Algae in Tropical Soils. In: Dommer-Gues, Y. and Diem, H., Eds., Microbiology of Tropical Soils and Plant Productivity, Martinus Nijhoff Publisher, La Hague, 147168.

[13] Issa, O.M., Defarge, C., Bissonnais, Y.L., Martin, B., Duval, A., Bruand, A., D’Acqui, L.P., Nodernberg, S. and Annerman, M. (2007) Effects of the Inoculation Cyanobacteria on the Microstructure and the Structural Stability of a Tropical Soil. Plant and Soil, 290, 209-219. http://dx.doi.org/10.1007/s11104-006-9153-9

[14] Roger, P.A. (1982) Blue Green Algae in Rice Fields. Proceedings of the Consultants Meeting, FAO/IAEA Joint Project, Vienna, 11-15 October 1982, 99-117.

[15] John, K.C. (1963) Species of Algae Found in Acid Sulphur Springs of Kerala. Agricultural Research Journal of Kerala, 2, 20-21.

[16] Aiyer, R.S (1965) Comparative Algological studies in rice fields in Kerala State. Agricultural Research Journal of Kerala, 3, 100-104.

[17] Anand, N. and Hopper, R.S.S. (1987) Blue Green Algae from Rice Fields in Kerala State, India. Hydrobiologia, 144, 223-232. http://dx.doi.org/10.1007/BF00005556

[18] Anand, N. and Hopper, R.S.S. (1995) Distribution of Blue Green Algae from Rice Fields of Kerala State, India. Phykos, 34, 55-64.

[19] Amma, P.A., Aiyer, R.S. and Subramoney, N. (1966) Occurrence of Blue Green Algae in Acid Soils of Kerala. Agricultural Research Journal of Kerala, 4, 141-143.

[20] Dominic, T.K. and Madhusoodanan, P.V. (1999) Cyanobacteria from Extreme Acidic Environments. Current Science, 77, 1021-1022.

[21] Sudheesh, T. and Shima, A. (2011) Effect of Organic Content on Geotechnical Properties of Kuttanad Clay. Electronic Journal of Geotechnical Engineering, 16, 1653-1663.

[22] Pillai, V.K., Ponniah, A.G., Vincent, D. and David, R. (1983) Acidity in Vembanadu Lake Causes Fish Mortality. Marine Fisheries Information Service-Technical and Extension Series, No. 53.

[23] Ray, J.G., Dhanya, V. and Binoy, T.T. (2014) Globally Unique Kuttanadu Wetland Paddy Soil of South India: Soil Fertility in Relation to Seasons and Different Stages of Crop. International Journal of Agriculture Photon, 125, 296304.

[24] Desikachary, T.V. (1959) Cyanophyta. ICAR Monograph on Algae, New Delhi, 1-686.

[25] Guiry, M.D. and Guiry, G.M. (2012) Worldwide Electronic Publication. National University of Ireland, Gal-Way. http://www.algaebase.org

[26] Dhanya, V. and Ray, J.G. (2015) Green Algae of Unique Tropical Wetland, Kuttanadu, Kerala, India, in Relation to Soil Regions, Seasons and Paddy Growth Stages. International Journal of Science, Environment and Technology, 4, 770-803.

[27] Prasanna, R., Lata, N., Radhika, A., Jadhav, S., Monica, J. and Brahma, D.K. (2009) Rhizosphere Dynamics of Inoculated Cyanobacteria and Their Growth-Promoting Role in Rice Crop. Egyptian Journal of Biology, 11, 26-36.

[28] Choudhary, K.K. and Bimal, R. (2010) Distribution of Nitrogen Fixing Cyanobacteria (Nostocaceae) during Rice Cul- 
tivation in Fertilized and Unfertilized Paddy Fields. Nordic Journal of Botany, 28, 100-103. http://dx.doi.org/10.1111/j.1756-1051.2009.00486.x

[29] Zancan, S., Trevisan, R. and Paoletti, M.G. (2006) Soil Algae Composition under Different Agro-Ecosystem in NorthEastern Italy. Agriculture, Ecosystems \& Environment, 112, 1-12. http://dx.doi.org/10.1016/j.agee.2005.06.018

[30] Vijayakumar, S., Thajuddin, N. and Manoharan, C. (2007) Biodiversity of Cyanobacteria in Industrial Effluents. Acta Botanica Malacitana, 32, 27-34.

[31] Bongale, U.D. (1981) Abundance of Algal Species in Relation to Soil Types and Major Crops in Cultivated Soils of Karnataka State, India. Plant and Soil, 60, 289-293. http://dx.doi.org/10.1007/BF02374112

[32] El-Gamal, A.D., Nady, A., Ghanem, E., Eisha, Y.E., Ayouty, I. and Shehatha, E.F. (2008) Studies on Soil Algal Flora in Kafrel-Sheikh Governorate Egypt. Egyptian Journal of Phycology, 9, 1-23.

[33] Lukesova, A. (2001) Soil Algae in Brown coal and Lignite Post mining areas in Central Europe(Czech Republic and Germany). Restoration Ecology, 9, 341-350. http://dx.doi.org/10.1046/j.1526-100X.2001.94002.x

[34] Singh, P.K., Kishore, S., Prakash, S. and Singh, K. (2008) Cyanophycean Algae Inhabiting Sodic Soil Exhibit Diverse Morphology: An Adaptation to High Exchangeable Sodium. Ecoprint, 15, 15-21.

[35] Broady, P.A. (1979) Quantitative Studies on the Terrestrial Algae of Signy Island, South Orkney Islands. British Antarctic Survey Bulletin, 47, 31-41.

[36] Mataloni, G., Tell, G. and Wynn-Williams, D.D. (2000) Structure and Diversity of Soil Algal Communities from Cierva Point (Antarctic Peninsula). Polar Biology, 23, 205-211. http://dx.doi.org/10.1007/s003000050028

[37] Jayabhaye, U.M. (2010) Studies on Phytoplankton Diversity in Sawana Dam, Maharashtra India. Shodh Samikshaaur Mulyankan, 2, 40-42.

[38] Irissari, P., Susan, G. and Monza, J. (2001) Cyanobacteria in Uruguayan Rice Fields: Diversity, Nitrogen Fixing Ability and Tolerance to Herbicides and Combined Nitrogen. Journal of Biotechnology, 91, 95-103. http://dx.doi.org/10.1016/S0168-1656(01)00334-0

[39] Choudhary, K.K. (2011) Occurrence of Nitrogen Fixing Cyanobacteria during Different Stages of Paddy Cultivation. Bangladesh Journal of Plant Taxonomy, 18, 73-76. http://dx.doi.org/10.3329/bjpt.v18i1.7842

[40] Metting, B. (1981) The Systematic and Ecology of Soil Algae. The Botanical Review, 147, 195-312.

[41] Horne, A.J. and Goldman, C.R. (1972) Nitrogen Fixation in Clear Lake, California 1. Seasonal Variation and the Role of heterocysts. Limnology and Oceanography, 17, 678-692. http://dx.doi.org/10.4319/lo.1972.17.5.0678

[42] Hoyos, C., Negro, A.I. and Aldasor, J.J. (2004) Cyanobacteria Distribution and Abundance in the Spanish Water Reservoirs during the Mal Stratification. Limnetica, 23, 119-132.

[43] Comerton, M. and Houghton, J.A. (1978) The Effects of Fertilizers on the Algal Flora of Peat. Proceedings of the Royal Irish Academy, 8, 233-245.

[44] Ohtani, S., Kousuke, S., Yamamoto, H., Aridomi, Y., Ritsuko, I. and Fukuoka, Y. (2000) Distribution of Soil Algae at the Monitoring Sites in the Vicinity of Syowa Station between Austral Summers of 1992/1993 and 1997/1998. Polar Bioscience, 13, 113-132.

[45] Syiem, M.B. (2005) Entrapped Cyanobacteria: Implications for Biotechnology. Indian Journal of Biotechnology, 4, 209-215. 